\title{
Transferencia de Calor y Materia durante la Fritura de Trozos de Ñame (Dioscórea alata)
}

\section{Armando Alvis ${ }^{1 *}$, Luis E. Cortés ${ }^{2}$ y Manuel Páez ${ }^{3}$}

(1) Universidad de Córdoba, Facultad de Ciencias Agrícolas, Departamento de Ingeniería de Alimentos, Carrera 6 No 76-103, Km. 3, vía Cereté, Córdoba-Colombia (e-mail: aalvis@hotmail.com) (2) Universidad Nacional de Colombia, Sede Palmira, Facultad de Ingeniería y Administración, Departamento de Ciencias Básicas, Carrera 32 Chapinero Vía Candelaria, Palmira-Colombia

(3) Universidad de Córdoba, Facultad de Ciencias Básicas e Ingeniería, Departamento de Química, Carrera 6 No 76-103, Km. 3, vía Cereté, Córdoba-Colombia

*autor a quien debe ser dirigida la correspondencia

\section{Resumen}

En el presente trabajo se analizó la transferencia de calor y materia durante el freído de trozos de ñame en aceite de soya a $140,160,180^{\circ} \mathrm{C}$. Los coeficientes de transferencia de calor y materia fueron determinados a partir de las gráficas de las relaciones adimensionales de temperatura y concentración de humedad versus tiempo. Los coeficientes de transferencia de calor y materia se incrementaron al aumentar la temperatura del aceite. Para la variedad de ñame llamada Pico de Botella, la pérdida de humedad durante el freído por inmersión a distintos tiempos disminuyó con el aumento de la temperatura del aceite. Se encontraron valores del coeficiente de difusión de humedad de $1.95,2.59$ y $3.24 \times 10^{-9} \mathrm{~m}^{2} / \mathrm{s}$, para las tres temperaturas de freído estudiadas.

Palabras clave: transferencia de calor y materia, coeficiente de difusión, freído por inmersión, ñame

\section{Heat and Mass Transfer during the Frying of Slices of Yam (Dioscórea alata)}

\begin{abstract}
Heat and mass transfer during frying slices of yam in soybean oil at $140,160,180{ }^{\circ} \mathrm{C}$ were studied. Heat and mass transfer coefficients were determined from the relation between dimensionless temperature and moisture concentration and time, respectively. The heat and mass transfer coefficients increased as the temperature of the oil increased. For the variety of yam known as Pico de Botella, moisture loss during deep fat frying at different times decreased with increasing temperature of the oil. Values of diffusion coefficient of moisture were 1.95, 2.59 and $3.24 \times 10^{-9}$ $\mathrm{m}^{2} / \mathrm{s}$, for the frying temperatures considered in the study.
\end{abstract}

Key words: heat and mass transfer, diffusion coefficient, deep fat frying, yam 


\section{INTRODUCCION}

El ñame (Dioscórea alata) es un alimento básico en muchas regiones tropicales, principalmente en el occidente de África, Asia y el Caribe (FAO, 1990). En esta última región, en la Costa Atlántica colombiana, se cultivan alrededor de 29757 hectáreas, que involucran a 9000 familias de pequeños productores con un área promedio de 1.4 hectáreas por familia y un rendimiento promedio de 11.9 Ton/ha y con una producción de 264500Ton/año (Minagricultura, 2003).

Cuando un alimento se sumerge en un medio caliente tal como aceite su temperatura aumenta rápidamente y el agua que contiene se elimina en forma de vapor, por lo que su superficie empieza a deshidratarse, se forma una corteza y el frente de evaporación va trasladándose hacia el interior del producto (Fellows, 1994, Moreira et al., 1999). La temperatura en la superficie del alimento alcanza la del fluido caliente y la interna aumenta lentamente hasta alcanzar los $100{ }^{\circ} \mathrm{C}$ (Fellows, 1994). Las velocidades de transferencia de calor al alimento dependen de la diferencia de temperaturas entre éste y el fluido y del coeficiente de conductancia térmica superficial. La velocidad a la que el calor penetra hacia el interior del material, depende de la conductividad térmica. Las características de la fritura están íntimamente relacionadas con las propiedades fisicoquímicas y funcionales de los constituyentes de los alimentos, así como las variables de operación que se aplican en las diferentes etapas del proceso (Moreira et al., 1999). La interacción de los fenómenos de transferencia de calor y masa hace que la fritura sea una de las operaciones unitarias más difíciles de entender en la cual el coeficiente de transferencia de calor por convección juega un papel preponderante (Whitaker, 1977; Singh, 1995; Farkas y Hubbard, 2000).

Para el modelado y cálculo de sistemas de freído de alimentos por inmersión en aceite es muy importante la determinación de los coeficientes de transferencia de calor y masa. Los métodos reportados en la literatura para medir dicho coeficiente presentan algunas limitaciones (Moreira et al., 1999) tales como: a) Faltan técnicas estándar de medida, b) Pocas veces se toma en consideración la transferencia de masa, c) Escaso conocimiento del coeficiente de transferencia de calor (en función de la posición del material), d) No existe uniformidad en la estimación y reporte de errores de medida, e) Geometría del producto. Costa et al. (1999), usaron un método indirecto (una pieza de metal con la misma geometría de una papa a la inglesa) y un método directo (papa a la francesa) para calcular el coeficiente de transferencia de calor.

Sahin et al. (1999), utilizaron el método de temperatura en estado transitorio para determinar el coeficiente de transferencia de calor de un paralelepípedo de aluminio relleno con masa de papa sumergido en aceite de girasol a las temperaturas de 150 y $190{ }^{\circ} \mathrm{C}$. Encontraron valores de $h$ entre 90 y $200 \mathrm{~W} / \mathrm{m}^{2} \mathrm{~K}$. Hubbard y Farkas (2000a), usaron cilindros de papa para determinar el coeficiente de transferencia de calor por convección durante el freído por inmersión a 120, 150 y $180{ }^{\circ} \mathrm{C}$, Los valores del coeficiente de transferencia de calor fueron de 610,650 y $890 \mathrm{~W} / \mathrm{m}^{2} \mathrm{~K}$.

Vélez y Sosa-Morales (2003), usaron donas de forma cilíndrica de anillo interior 1,5 cm. y anillo exterior 2,0 cm. para determinar el coeficiente de transferencia de calor durante el freído por inmersión a temperaturas de 180,190 y $200{ }^{\circ} \mathrm{C}$, los valores del coeficiente de transferencia de calor fueron de 158,$63 ; 269,54$ y $296,84 \mathrm{~W} / \mathrm{m}^{2} \mathrm{~K}$.

Sosa-Morales et al. (2006), estudiaron simultáneamente la transferencia de calor y masa y la evolución de las propiedades físicas ( $C p, k, \alpha$ y $\rho$ ) durante el freído por inmersión de carne de cerdo en forma de placa de $5 \times 4 \times 2 \mathrm{~cm}$., para lo cual usaron aceite de girasol a temperaturas de 90,100 y $110{ }^{\circ} \mathrm{C}$ y lo compararon con la fritura en grasa de pastelería a $100{ }^{\circ} \mathrm{C}$. Los autores encontraron coeficientes de transferencia de calor entre 187.7 a $226.1 \mathrm{~W} / \mathrm{m}^{2}{ }^{\circ} \mathrm{C}$ y valores de difusividad de masa entre 1.5 y $30.2 \times 10^{-9} \mathrm{~m}^{2} / \mathrm{s}$.

\section{MATERIALES Y METODOS}

Para la determinación del coeficiente de transferencia de calor se utilizaron trozos de ñame en forma de paralelepípedo de $0.8 \times 0.8 \times 4 \mathrm{~cm}$ cortados en una trozadora de papa. Las condiciones de proceso fueron temperatura de fritura de 140,160 y $180^{\circ} \mathrm{C}$ y tiempos de freído de 5 a $180 \mathrm{~s}$, con 
variaciones de $5 \mathrm{~s}$. Se utilizó para ello aceite de soya y una freidora MARCA MKE (Indianápolis EUA) de $5 \mathrm{I}$. de capacidad, equipada con un termostato con precisión de $\pm 0.1{ }^{\circ} \mathrm{C}$.

Para medir la variación de temperatura de los trozos se insertaron 2 termopares tipo $\mathrm{K}$ de 0,25 mm de diámetro por los extremos del trozo en sentido longitudinal hasta que las puntas de los dos termopares estuvieran cerca del centro geométrico (aproximadamente $3.5 \mathrm{~mm}$ ) y suficientemente lejos de los extremos Figura 1. Otro termopar de las mismas características se usó para medir la temperatura del aceite. Para cada condición de freído se utilizaron 2 trozos de ñame y se realizaron tres réplicas.

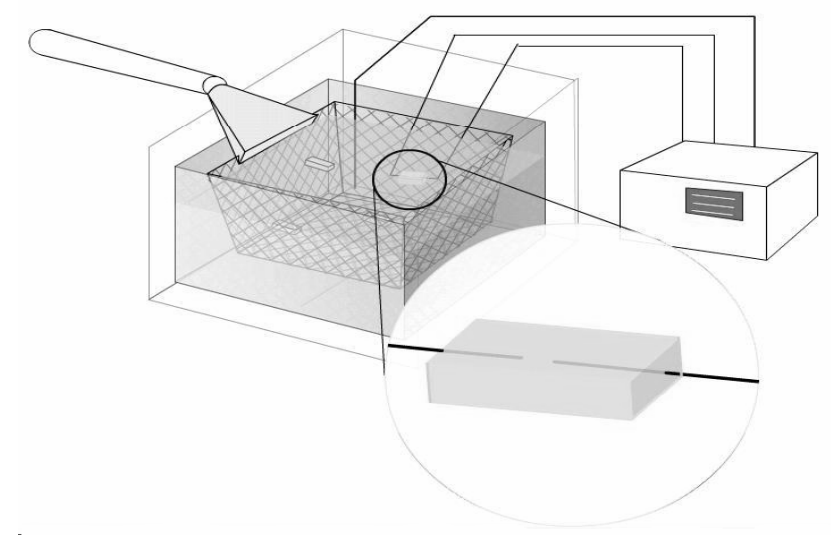

Fig. 1: Esquema del experimento de freído por inmersión.

Las temperaturas se registraron cada segundo por medio de un sistema adquisición de datos acoplado a un computador personal (Figura 2).

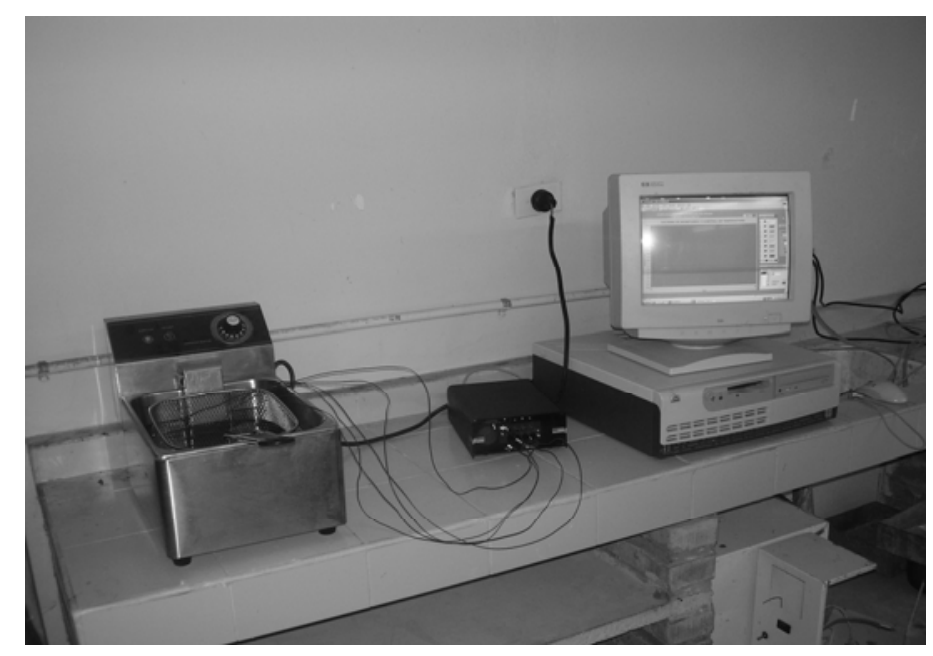

Fig. 2: Sistema de adquisición de datos del experimento de freído.

El coeficiente de transferencia de calor se determinó a partir de la solución analítica de la ecuación diferencial de conducción de calor en una dimensión en coordenadas cartesianas para una placa infinita de espesor 2L, ecuación (1) empleando las condiciones límites y la condición inicial presentadas en la ecuación (2)

$$
\begin{aligned}
& \frac{\partial^{2} T}{\partial x^{2}}=\frac{1}{\alpha} \frac{\partial T}{\partial t}, \quad 0 \leq x \leq L \\
& \frac{\partial T}{\partial x}=0 \quad x=0 ;-k \frac{\partial T}{\partial x}=h\left(T-T_{\infty}\right) \quad x=L \quad T=T_{i} \quad t=0
\end{aligned}
$$


Donde: $T$, temperatura $\left({ }^{\circ} \mathrm{C}\right), T_{i}$, temperatura inicial del producto $\left({ }^{\circ} \mathrm{C}\right), T_{\infty}$, temperatura del aceite $\left({ }^{\circ} \mathrm{C}\right), k$, conductividad térmica del producto $\mathrm{W} / \mathrm{m}{ }^{\circ} \mathrm{C}, x$, localización, donde la temperatura es medida en el infinito de la lámina $0 \leq x \leq L, t$, tiempo (s), $L$, mitad del espesor $(\mathrm{m}), h$, coeficiente de transferencia de calor $\left(\mathrm{W} / \mathrm{m}^{2}{ }^{\circ} \mathrm{C}\right)$.

La siguiente solución de serie infinita muestra la temperatura localizada en cualquier punto dentro de la lámina como una función del tiempo:

$$
\left(\frac{T(x, t)-T_{\infty}}{T_{i}-T_{\infty}}\right)=\sum_{n=1}^{\infty} \frac{2 \operatorname{Sen} \delta_{n}}{\delta_{n}+\left(\operatorname{Sen} \delta_{n}\right)\left(\operatorname{Cos} \delta_{n}\right)} \operatorname{Cos}\left(\delta_{n} \frac{x}{L}\right) e^{\left(-\delta_{n}^{2} \frac{\alpha t}{L^{2}}\right)}
$$

Para números de Fourier $\left(\frac{\alpha t}{L^{2}}\right)$ mayor que 0.1 el primer término de la ecuación (3) provee resultados con suficiente precisión (Crank, 1975). La forma reducida de la ecuación (3) puede ser usada para obtener la solución para trozos de ñame en forma de paralelepípedo (finito en dos dimensiones), haciendo uso de la regla de superposición, según la cual, la solución para la transferencia de calor de una lámina finita en 2 dimensiones se encuentra multiplicando la solución para 2 láminas infinitas, ecuación (4), desarrollada por Newman en 1936 (Geankoplis, 1993).

$$
\frac{\theta}{\theta_{i}}=\left(\frac{T(x, y, t)-T_{\infty}}{T_{i}-T_{\infty}}\right)_{\text {Láminafinita }}=\left(\frac{T(x, t)-T_{\infty}}{T_{i}-T_{\infty}}\right)_{\text {Láminainf inita } 2 L}\left(\frac{T(y, t)-T_{\infty}}{T_{i}-T_{\infty}}\right)_{\text {Láminainf inita } 2 L}
$$

Donde: $T(x, y, t)$ temperatura en cualquier punto y en cualquier tiempo, $\left({ }^{\circ} \mathrm{C}\right)$. Reemplazando en (4) para placas infinitas de espesor finito en $x$ y $y$, se obtienen las ecuaciones (5) y (6).

$$
\begin{aligned}
& \left(\frac{T(x, t)-T_{\infty}}{T_{i}-T_{\infty}}\right)=\frac{2 \operatorname{Sen} \delta_{n}}{\delta_{n}+\left(\operatorname{Sen} \delta_{n}\right)\left(\operatorname{Cos} \delta_{n}\right)} \operatorname{Cos}\left(\delta_{n} \frac{x}{L}\right) e^{\left(-\delta_{n}^{2} \frac{\alpha t}{L^{2}}\right)} \\
& \left(\frac{T(y, t)-T_{\infty}}{T_{i}-T_{\infty}}\right)=\frac{2 \operatorname{Sen} \delta_{n}}{\delta_{n}+\left(\operatorname{Sen} \delta_{n}\right)\left(\operatorname{Cos} \delta_{n}\right)} \operatorname{Cos}\left(\delta_{n} \frac{y}{L}\right) e^{\left(-\delta_{n}^{2} \frac{\alpha t}{L^{2}}\right)}
\end{aligned}
$$

Y al hacer el producto de las dos anteriores resulta:

$$
\left(\frac{T(x, t)-T_{\infty}}{T_{i}-T_{\infty}}\right)\left(\frac{T(y, t)-T \infty}{T_{i}-T_{\infty}}\right)=A e^{\left(-\delta_{n}^{2} \frac{\alpha t}{L^{2}}\right)}
$$

Donde:

$$
A=\left(\frac{2 \operatorname{Sen} \delta_{n}}{\delta_{n}+\left(\operatorname{Sen} \delta_{n}\right)\left(\operatorname{Cos} \delta_{n}\right)}\right)^{2} \cos \left(\delta_{n} \frac{x}{L}\right) \cos \left(\delta_{n} \frac{y}{L}\right)
$$

Linealizando la ecuación (7) resulta la ecuación de una recta

$$
\ln \left(\frac{\left(T(x, y, t)-T_{\infty}\right.}{T_{i}-T_{\infty}}\right)=\ln A-2 \delta_{n}^{2} \frac{\alpha t}{L^{2}}
$$

Si se grafica $\ln \left(\frac{T(x, y, t)-T_{\infty}}{T_{i}-T_{\infty}}\right)$ vs $t$ la pendiente de la sección lineal de esta gráfica es igual a . $-2 \delta_{n}^{2} \frac{\alpha t}{L^{2}}$. Conocidos $\alpha$ : Difusividad térmica $\left(\mathrm{m}^{2} / \mathrm{s}\right)$ y $L:$ mitad del espesor en $(m)$ se determina $\delta_{n}$. Una vez hallado $\delta_{n}$, se determina Bi y posteriormente $h$, así: 


$$
\begin{aligned}
& B i=\delta_{n} \operatorname{Tan} \delta_{n} \\
& B i=\frac{h L}{k}
\end{aligned}
$$

La conductividad, la difusividad térmica, el calor específico y la densidad de la muestra fueron determinados con base en la composición del alimento y la temperatura con las fórmulas sugerida por (Choi y Okos, 1985), por lo que fue necesario calcular el contenido de humedad, grasa, ceniza, fibra, proteínas del ñame frito a las condiciones de proceso. Temperatura de 140,160 y $180{ }^{\circ} \mathrm{C}$ y tiempos de 1 a 9 minutos. La conductividad térmica fue de $0.4401 \mathrm{~W} / \mathrm{m}^{\circ} \mathrm{C}$ y la difusividad $1.23^{\star} 10^{-7}$ $\mathrm{m}^{-2} / \mathrm{s}$.

Inicialmente, para la determinación del coeficiente de transferencia de masa a diferentes temperatura de fritura $\left(140,160\right.$ y $\left.180^{\circ} \mathrm{C}\right)$ se sumergieron trozos de ñame $(0.8 \times 0.8 \times 4 \mathrm{~cm}$.) en aceite refinado de soya en una freidora de acero inoxidable de $5 \mathrm{I}$. de capacidad. Al cabo de diferentes tiempos (10, $20,30,40,60,80,100,120,180$, y de ahí en adelante cada 30 segundos hasta 420 s) se sacaron muestras y se colocaron en papel de filtro para remover el aceite superficial e inmediatamente se les determinó la humedad a $105^{\circ} \mathrm{C}$ hasta peso constante (A.O.A.C., 2003) y el contenido de aceite por extracción por solvente con hexano por 8 h. (A.O.A.C., 2003). Para cada temperatura de freído se realizaron tres replicas.

Los datos de contenido de humedad de los trozos en función del tiempo se utilizaron posteriormente en un método matemático para la determinación del coeficiente de transferencia de masa, de manera análoga al método usado para transferencia de calor. Se utilizó para ello la ecuación de concentración en función del tiempo y la posición, para una lámina infinita de espesor 2L, que se obtiene resolviendo la ecuación diferencial de difusión en estado transitorio (12), con las condiciones límites e inicial mostrados en (13).

$$
\begin{aligned}
& \frac{\partial C}{\partial x^{2}}=\frac{1}{D} \frac{\partial C}{\partial t} \quad 0 \leq x \leq L \quad \text { para } t>0 \\
& \frac{\partial C}{\partial x}=0 \text { en } x=0 ;-\mathrm{D} \frac{\partial C}{\partial x}=k_{c}\left(C-C_{\infty}\right) \text { en } x=L ; C=C_{i} \text { cuando } t=0
\end{aligned}
$$

La siguiente solución como serie infinita muestra la concentración de la humedad localizada en cualquier punto dentro de la lámina como una función del tiempo.

$$
\left(\frac{C(x, t)-C_{\infty}}{C_{i}-C_{\infty}}\right)=\sum_{n=0}^{n=\infty} \frac{2 \operatorname{Sen} \delta_{n}}{\delta_{n}+\left(\operatorname{Sen} \delta_{n}\right)\left(\operatorname{Cos} \delta_{n}\right)} \operatorname{Cos}\left(\delta_{n} \frac{x}{L}\right) e^{\left(-\delta_{m}^{2} \frac{D t}{L^{2}}\right)}
$$

La forma reducida de la ecuación (14) puede ser usada para obtener la solución para trozos de ñame en forma de paralelepípedo (finito en dos dimensiones), haciendo uso de la regla de superposición, según la cual, la solución para la transferencia de masa de una lámina finita en 2 dimensiones se encuentra multiplicando la solución para 2 láminas infinitas, ecuación (15), desarrollada por Crank (1975).

$$
\frac{C}{C_{i}}=\left(\frac{C(x, y, t)-C_{\infty}}{C_{i}-C_{\infty}}\right)_{\text {Láminafinita }}=\left(\frac{C(x, t)-C_{\infty}}{C_{i}-C_{\infty}}\right)_{\text {Láminainf inita } 2 L}\left(\frac{C(y, t)-C_{\infty}}{C_{i}-C_{\infty}}\right)_{\text {Láminainf inita } 2 L}
$$

Donde: $\mathrm{C}(\mathrm{x}, \mathrm{y}, \mathrm{t})$ Concentración de humedad en cualquier punto y en cualquier tiempo, (kg /kg sólidos)

$$
\left(\frac{C(x, t)-C_{\infty}}{C_{i}-C_{\infty}}\right)=\frac{2 \operatorname{Sen} \delta_{n}}{\delta_{n}+\left(\operatorname{Sen} \delta_{n}\right)\left(\operatorname{Cos} \delta_{n}\right)} \operatorname{Cos}\left(\delta_{n} \frac{x}{L}\right) e^{\left(-\delta_{n}^{2} \frac{D_{a} t}{L^{2}}\right)}
$$


$\left(\frac{C(y, t)-C_{\infty}}{C_{i}-C_{\infty}}\right)=\frac{2 \operatorname{Sen} \delta_{n}}{\delta_{n}+\left(\operatorname{Sen} \delta_{n}\right)\left(\operatorname{Cos} \delta_{n}\right)} \operatorname{Cos}\left(\delta_{n} \frac{y}{L}\right) e^{\left(-\delta_{n}^{2} \frac{D_{a} t}{L^{2}}\right)}$

El producto de (16) por (17) da como resultado (18)

$\left(\frac{C(x, t)-C_{\infty}}{C_{i}-C_{\infty}}\right)\left(\frac{C(y, t)-C_{\infty}}{C_{i}-C_{\infty}}\right)=E e^{\left(-\delta_{n}^{2} \frac{\alpha t}{L^{2}}\right)}$

Donde:

$E=\frac{2 \operatorname{Sen}^{2} \delta_{n}}{\delta_{n}\left(\delta_{n}+\operatorname{Sen} \delta_{n} \operatorname{Cos} \delta_{n}\right)}$

Linealizando la ecuación (18),

$\ln \left(\frac{\left(C(x, y, t)-C_{\infty}\right.}{C_{i}-C_{\infty}}\right)=\ln E-2 \delta_{n}^{2} \frac{D_{a} t}{L^{2}}$

Si se grafica $\ln \left(\frac{C(x, y, t)-C_{\infty}}{C_{i}-C_{\infty}}\right)$ vs t., desde el intercepto de la grafica fue calculada la primera raíz de la ecuación característica $\left(\delta_{n}\right)$. Después se determinó el coeficiente de difusión, $D_{a},\left(\mathrm{~m}^{2} / \mathrm{s}\right)$ desde la pendiente de la sección lineal de esta grafica que se iguala a $-2 \delta_{n}^{2} \frac{D_{a} t}{L^{2}}$.

Una vez hallado $\delta_{n}$, se determina $B i_{m}$ y posteriormente $k_{c}$.

$B i_{m}=\delta_{n} \operatorname{Tan} \delta_{n}$

$B i_{m}=\frac{k_{c} L}{D_{a}}$

\section{ANALISIS DE RESULTADOS}

En la Figura 3 se muestra la variación de la temperatura de los trozos de ñame con el tiempo para diferentes temperaturas de freído, observándose que en el interior del producto la temperatura aumentó rápidamente al comienzo del proceso, estabilizándose en 103 a $104{ }^{\circ} \mathrm{C}$ a partir de $70-80$ segundos, dependiendo de la temperatura del aceite. Esto puede ser atribuido a que el interior del producto aun no estaba lo suficientemente seco para permitir el incremento de la energía interna. Cuando la temperatura interna alcanza el punto de ebullición del agua la vaporización mantiene la temperatura constante hasta que el agua está casi totalmente evaporada. Este fenómeno fue observado por Claeys en 2005 (Yildiz et al., 2007). Según estos autores, las temperaturas arriba del punto de ebullición del agua sólo son alcanzadas cuando el alimento esta casi seco. Como en el caso de fritura de papa reportado por Budzaki y Seruga (2005), el punto de ebullición del agua dentro del ñame frito fue levemente más alto que el punto de ebullición del agua pura. Los autores mencionados justifican que esto puede deberse a la presencia de solutos disueltos.

Los diagramas de la relación de temperatura adimensional vs el tiempo obtenido a las temperaturas de 140, 160 y $180{ }^{\circ} \mathrm{C}$ freído son presentados en la Figura 4. Como se observa en la figura, la velocidad de calentamiento de los trozos de ñame decrece con el incremento de la temperatura de freído. Las pendientes de la sección lineal de estas curvas Figura 5 se obtuvieron mediante análisis de regresión lineal y fueron usadas posteriormente para determinar el coeficiente de transferencia de calor efectivo. 

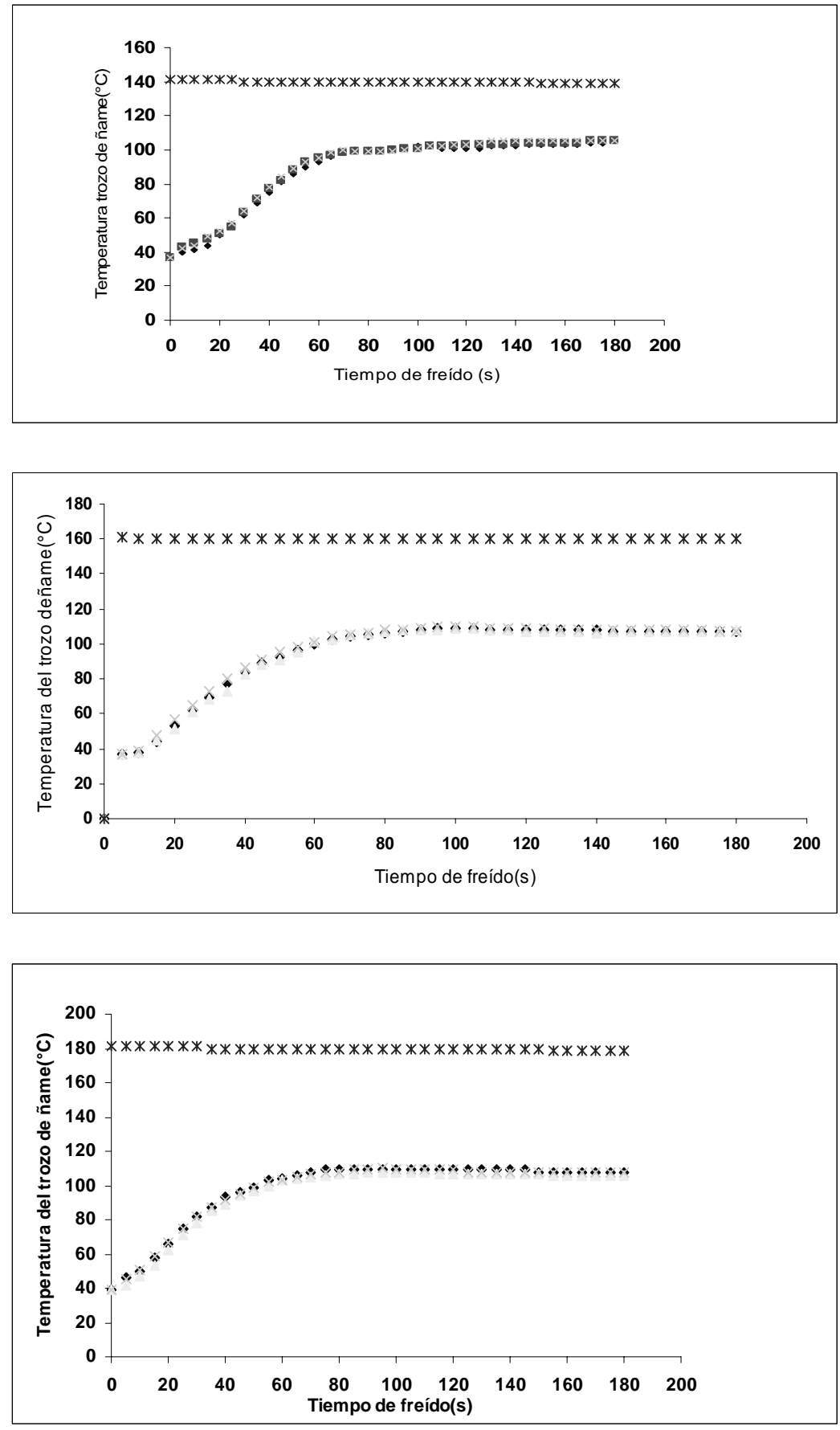

Fig. 3: Temperatura del trozo de ñame durante el freído por inmersión a la temperatura del aceite, 140,160 y $180^{\circ} \mathrm{C}$.

Los valores del número de Biot necesarios para el cálculo del coeficiente de transferencia de calor (ecuaciones 10 y 11) se tomaron de los tramos de mayor pendiente de las curvas de la Figura 5, situación que se observó en el período entre 15 y 35 segundos, en el cual la velocidad de calentamiento es mayor para las temperaturas de freído utilizadas. En la tabla 1 se muestran los valores calculados del número de Biot y el coeficiente de transferencia de calor con sus correspondientes desviaciones estándar.

Los valores del coeficiente de transferencia de calor encontrados en el presente trabajo para trozos de ñame (entre 148 y $204 \mathrm{~W} / \mathrm{m}^{2} \mathrm{~K}$ ) están dentro del rango de valores reportados por diferentes autores para otros productos vegetales (entre $90-1100 \mathrm{~W} / \mathrm{m}^{2} \mathrm{~K}$ ). Califano y Calvelo (1991), en el proceso de freído de papas reportaron valores de $\mathrm{h}$ (entre 150 y $165 \mathrm{~W} / \mathrm{m}^{2} \mathrm{~K}$ ) para temperaturas 
entre 50 y $100{ }^{\circ} \mathrm{C}$; Costa et al. (1999), encontraron valores de h a temperaturas entre 140 y $180{ }^{\circ} \mathrm{C}$, entre $594 \pm 38$ y $750 \pm 59 \mathrm{~W} / \mathrm{m}^{2} \mathrm{~K}$, para el método indirecto y entre $443 \pm 32$ y $650 \pm 7 \mathrm{~W} / \mathrm{m}^{2} \mathrm{~K}$, a las mismas temperaturas, para el método directo, para trozos de papa en forma de paralelepípedo freídos en aceite de girasol a temperaturas de 140 y $180{ }^{\circ} \mathrm{C}$; Hubbard y Farkas (2000b) usaron cilindros de papa, para determinar el coeficiente de transferencia de calor durante el freído por inmersión a $180^{\circ} \mathrm{C}$ y un tiempo de 200 a 900 segundos en la fase de ebullición y encontraron que $h$ era de 300 y $1100\left(W / m^{2} K\right)$; Budzaki y Seruga (2005), encontraron valores de h (entre 94.22 0.33 y $774.88 \pm 3.89 \mathrm{~W} / \mathrm{m}^{2} \mathrm{~K}$ ) para esferas formadas por una mezcla de harina de trigo y papa (50\% de cada una) al freírlas en aceite de soya a $190{ }^{\circ} \mathrm{C}$ durante $120 \mathrm{~s}$ y $5 \mathrm{~s}$, respectivamente. Según estos últimos autores, los valores mas altos del coeficiente de transferencia de calor se observaron en la etapa de ebullición y los valores mínimos, en la etapa de velocidad decreciente. Farinu y Baik (2007) trabajaron con esferas de batata sometidas a temperaturas de freído de 150, 160, 170 y $180{ }^{\circ} \mathrm{C}$ y encontraron valores de $\mathrm{h}$ (entre 710 y $837 \mathrm{~W} / \mathrm{m}^{2} \mathrm{~K}$ ). Yildiz et al. (2007), reportan valores efectivos de $\mathrm{h}$ entre 181 y $286\left(\mathrm{~W} / \mathrm{m}^{2} \mathrm{~K}\right)$ para paralelepípedos de papa freídos en aceite de girasol a temperaturas entre 150 y $190^{\circ} \mathrm{C}$.

Las diferencias entre los valores del coeficiente de transferencia de calor encontrados en el presente trabajo y los reportados en la literatura, pueden ser explicadas por la diferencia entre las características de los productos trabajados, los métodos utilizados para la medición de las temperaturas y el método de solución de las ecuaciones de transferencia de calor.

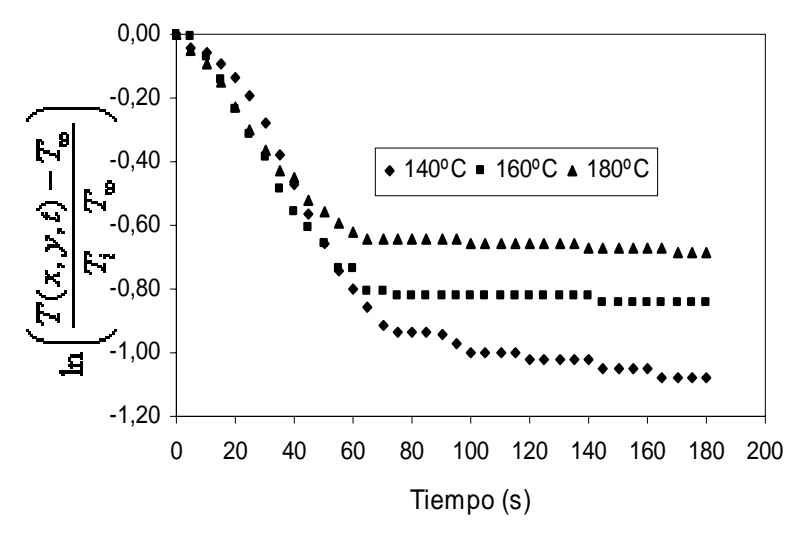

Fig.4: Relación adimensional de la temperatura vs el tiempo durante el experimento del freído por inmersión a diferentes temperaturas tiempo usada para la determinación del coeficiente de transferencia de calor.

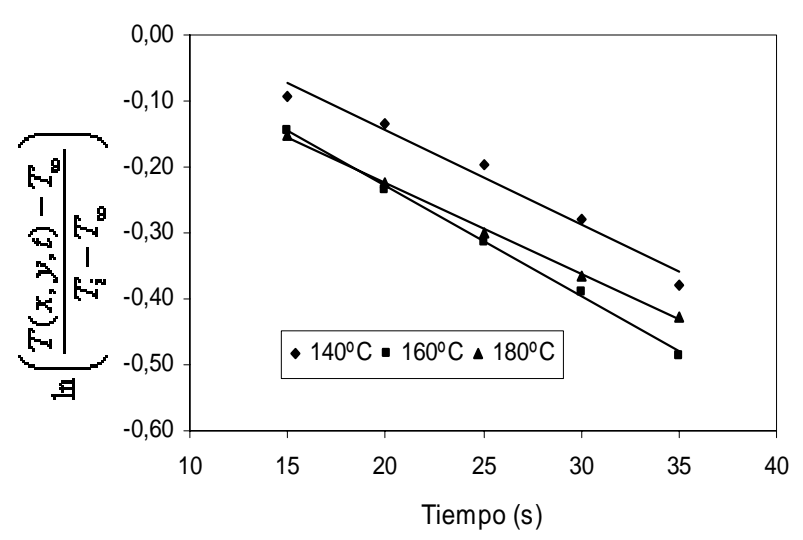

Fig. 5: Pendiente de la sección lineal de la relación adimensional de la temperatura vs el tiempo usada para la determinación del coeficiente de transferencia de calor

Tabla 1: Valores del número de Biot y el coeficiente de transferencia de calor en función de la temperatura del aceite.

\begin{tabular}{|c|c|c|}
\hline $\begin{array}{c}\text { Temperatura del aceite } \\
{ }^{\circ} \mathrm{C}\end{array}$ & $\begin{array}{c}\text { Número de Biot } \\
\mathrm{Bi}\end{array}$ & $\begin{array}{c}\text { Coeficiente de transferencia de calor } \\
\mathrm{W} / \mathrm{m}^{2}{ }^{\circ} \mathrm{C}\end{array}$ \\
\hline 140 & $1,24 \pm 0,09$ & $148,10 \pm 8,90$ \\
\hline 160 & $1,71 \pm 0,12$ & $192,08 \pm 10,6$ \\
\hline 180 & $1,86 \pm 0,34$ & $204,46 \pm 14,6$ \\
\hline
\end{tabular}

Los valores del coeficiente de difusión, fueron determinados desde la pendiente de la Figura 6 concentración adimensional vs el tiempo, y se muestran en la tabla 2, donde la velocidad de pérdida de humedad es mayor para las temperaturas de freído utilizadas. Los valores del coeficiente de difusión encontrados en el presente trabajo para trozos de ñame (entre $1.95^{\star} 10^{-9} \mathrm{~m}^{2} / \mathrm{s}$ y $3.24^{*} 10^{-9}$ $\mathrm{m}^{2} / \mathrm{s}$ ) son muy similares a los reportados por Yildiz et al. (2007) en trozos de papa, para los cuales obtuvieron valores de $9.2 \pm 1.1 * 10^{-9}, 11.0 \pm 1.0^{\star} 10^{-9}$ y $18.2 \pm 0.7 * 10^{-9} \mathrm{~m}^{2} / \mathrm{s}$ a las temperaturas de 150 , 
170 y $190{ }^{\circ} \mathrm{C}$ respectivamente; Moreira et al. (1995), para de tortillas de maíz $\left(9.34^{\star} 10^{-8} \mathrm{~m}^{2} / \mathrm{s}\right)$ y Sosa-Morales et al. (2006) para lonjas de carne de cerdo quienes obtuvieron valores de $1.59 * 10^{-9}$ $\mathrm{m}^{2} / \mathrm{s}, 9.15^{\star} 10^{-9} \mathrm{~m}^{2} / \mathrm{s}$ y $30.2^{\star} 10^{-9} \mathrm{~m}^{2} / \mathrm{s}$ a las temperaturas de 90,100 y $110^{\circ} \mathrm{C}$. Por otro lado, son menores que los reportados por Dincer y Yildiz (1996), para salchichas fritas a $180{ }^{\circ} \mathrm{C}\left(1.31 * 10^{-7}\right.$ $\mathrm{m}^{2} / \mathrm{s}$ ), pero mayores que algunos valores citados por Rice y Gamble (1989), para el freído de tajadas de papa a $145{ }^{\circ} \mathrm{C}\left(14\right.$ y $\left.24^{*} 10^{-10} \mathrm{~m}^{2} / \mathrm{s}\right)$ para los primeros 30 y $60 \mathrm{~s}$ respectivamente. Las diferencias pueden ser atribuidas a la naturaleza del producto, al proceso en particular (temperatura y tiempo) y a los métodos de determinación utilizados.

La pérdida de humedad fue mayor a altas temperaturas del aceite. Los diagramas de la relación de humedad adimensional vs el tiempo obtenido a las temperaturas de 140,160 y $180{ }^{\circ} \mathrm{C}$ son presentados en la Figura 6. Como se observa en la figura, la velocidad de pérdida de humedad de los trozos de ñame disminuye con el incremento de la temperatura de freído. Las pendientes de la sección lineal de estas curvas Figura 6, se obtuvieron mediante análisis de regresión lineal y fueron usadas posteriormente para determinar el coeficiente de transferencia de masa efectivo. Los valores del número de Biot necesarios para el cálculo del coeficiente de transferencia de masa (ecuaciones 21 y 22) se tomaron de los tramos de mayor pendiente de las curvas de la Figura 6, la sección lineal de las curvas a medida que pasaba el tiempo fueron usadas para determinar el coeficiente de transferencia de masa efectivo situación que se observó en el período 0 y 330 segundos, donde la velocidad de pérdida de humedad es mayor para las temperaturas de freído analizadas. En la tabla 2 se muestran los valores calculados del número de Biot $\left(\mathrm{Bi}_{\mathrm{m}}\right)$ y el coeficiente de transferencia de masa (kc) con sus correspondientes desviaciones estándar. Los valores del coeficiente de transferencia de masa encontrados en el presente trabajo para trozos de ñame, entre 0,786 $\pm 0,76$ y $3,312 \pm 1,04 * 10^{-6} \mathrm{~m} / \mathrm{s}$ están por debajo al reportado por Yildiz et al. (2007), para trozos de papa $\left(1.12 \pm 0.22 * 10^{-5} \mathrm{~m} / \mathrm{s}, 1.58 \pm 0.23^{*} 10^{-5}\right.$ y $\left.2.07 \pm 0.24^{*} 10^{-5} \mathrm{~m} / \mathrm{s}\right)$ freídos a temperaturas entre 150 y $190^{\circ} \mathrm{C}$ respectivamente, esto puede ser debido al método utilizado y al producto.

Tabla 2: Valores del número de Biot, coeficiente de transferencia de masa Difusividad de la humedad en función de la temperatura del aceite.

\begin{tabular}{cccc}
\hline $\begin{array}{c}\text { Temperatura } \\
\text { del aceite }{ }^{\circ} \mathrm{C}\end{array}$ & $\begin{array}{c}\text { Número de } \\
\text { Biot } \mathrm{B}_{\mathrm{i}}\end{array}$ & $\begin{array}{c}\text { Coeficiente de } \\
\text { transferencia de } \\
\text { masa } \\
\boldsymbol{k}_{\boldsymbol{c}} \boldsymbol{x} \mathbf{1} \mathbf{O}^{6} \mathbf{m} / \mathbf{s}\end{array}$ & $\begin{array}{c}\text { Coeficiente de } \\
\text { Difusividad }\end{array}$ \\
\hline 140 & $1,61 \pm 0,52$ & $0,78 \pm 0,76$ & $1 \mathbf{D}^{9} \mathbf{m}^{2} / \mathbf{s}$ \\
160 & $2,74 \pm 0,48$ & $1,77 \pm 0,93$ & 2,95 \\
180 & $4,08 \pm 0,98$ & $3,30 \pm 1,04$ & 3,24 \\
\hline
\end{tabular}

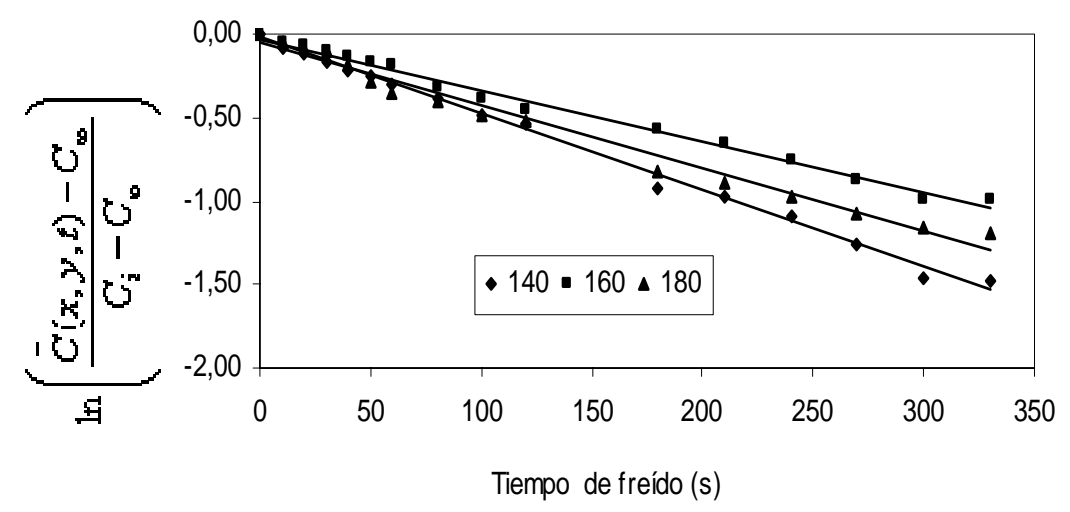

Fig. 6: Pendiente de la sección lineal de la relación adimensional concentración de la humedad vs el tiempo usada para la determinación del coeficiente de transferencia de masa 


\section{CONCLUSIONES}

El coeficiente de transferencia de calor $h$, aumentó al aumentarse la temperatura de freído. Los valores del coeficiente de transferencia de calor fueron $148.10 \pm 8.90,192.08 \pm 10.60$ y $204.46 \pm 14.6$ $\mathrm{W} / \mathrm{m}^{2}{ }^{\circ} \mathrm{C}$, a las temperaturas de 140,160 y $180{ }^{\circ} \mathrm{C}$, encontrándose dentro de los rangos reportados en la literatura por otros investigadores. El coeficiente de difusión para los trozos de ñame aumentó directamente con la temperatura de freído. Los valores obtenidos a 140,160 y $180{ }^{\circ} \mathrm{C}$ fueron 1.95 , 2.59 y $3.24 \times 10^{-9} \mathrm{~m}^{2} / \mathrm{s}$. El coeficiente de transferencia de masa $k c$, aumentó a medida que se incrementó la temperatura del aceite. Los valores del coeficiente de transferencia de masa fueron $7.86 \pm 0.761 * 10^{-7}, 1.771 \pm 0.933^{*} 10^{-6}$ y $3.312 \pm 1.04 * 10^{-6} \mathrm{~m} / \mathrm{s}$, a las temperaturas de $140,160 \mathrm{y}$ $180^{\circ} \mathrm{C}$.

\section{NOMENCLATURA}

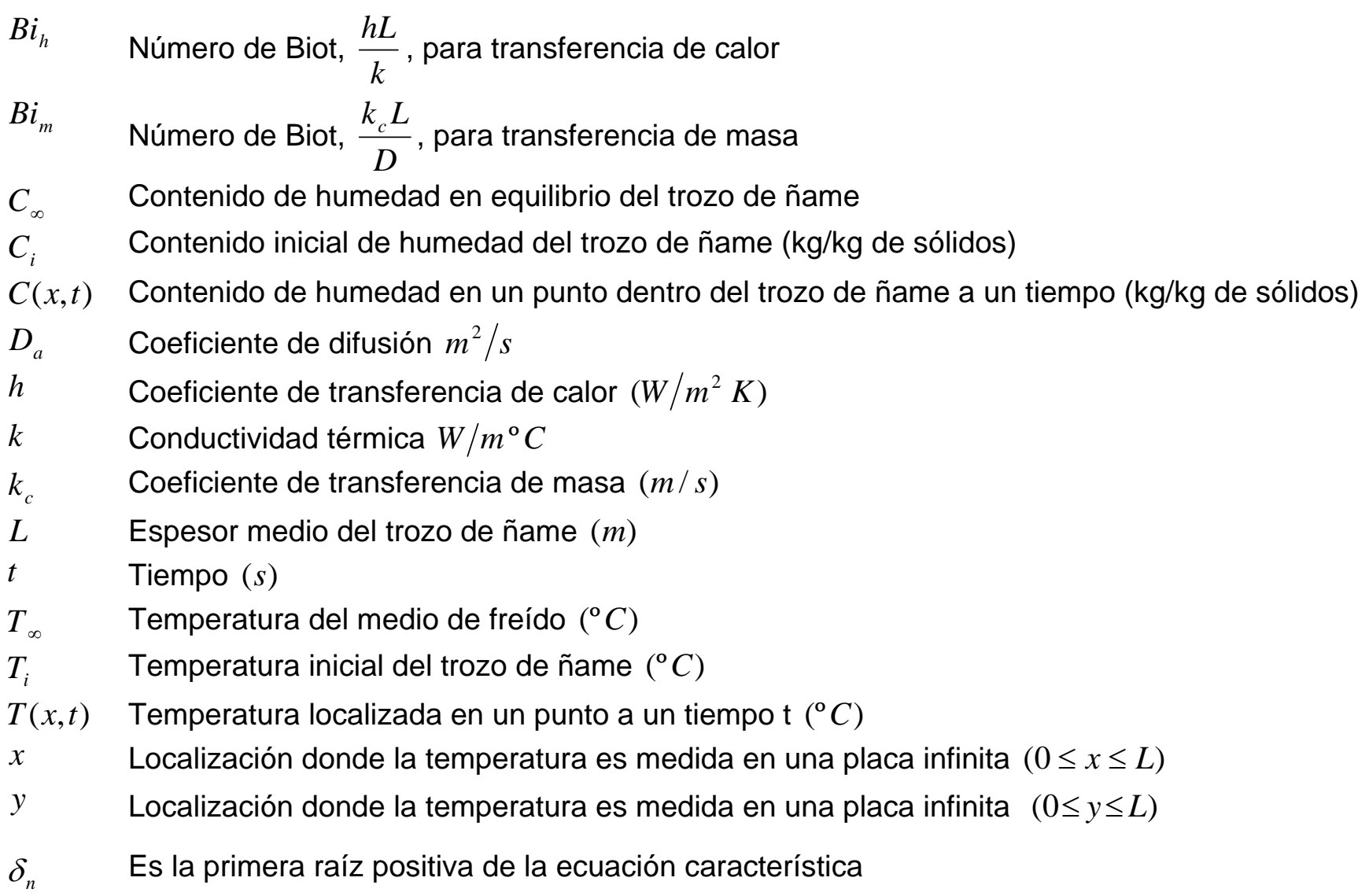

\section{REFERENCIAS}

A.O.A.C.; Association of Official Analytical Chemists, Official Methods of Analysis of AOAC International, 17th edition, Maryland, USA (2003).

Budzaki, S. y B. Seruga; Determination of convective heat transfer coefficient during frying of potato dough, Journal Food Engineering: 66(3), 307-314 (2005).

Califano, A.N. y A. Calvelo; Thermal conductivity of potato between 50 and $100{ }^{\circ} \mathrm{C}$. Journal of Food Science: 56, 586-589 (1991).

Choi, Y. y M.R. Okos, Thermal properties in liquid foods-Review. In: Physical and Chemical properties of food. Ed.M.Okos. American Society of Agricultural Engineering, p.p 93-101 (1985).

Costa, R.M., F.A.R., Oliveira, O. Delaney y V. Gekas; Analysis of the heat transfer coefficient during potato frying, Journal of Food Engineering: 39(3), 293-299 (1999). 
Crank. J.; The mathematics of diffusion. $2^{\text {nd }}$ Ed., Oxford University Press, London, pp. 69-88 (1975).

Dincer, I. y M. Yildiz; Modelling of thermal and moisture diffusions in cylindrically shaped sausages during frying, Journal of Food Engineering: 28, 35 -43 (1996).

FAO; Roots, tubers, plantains and bananas in human nutrition. FAO Food and Nutrition Series, $N^{\circ} 24$ (1990).

Farinu, A. y O.D. Baik; Heat transfer coefficients during deep fat frying of sweetpotato: Effects of product size and oil temperature, Food Research International: 40, 989-994 (2007).

Farkas, B. E. y L.J. Hubbard; Analysis of convective heat transfer during immersion frying drying. Journal of Food Technology: 18(5), 145 -151 (2000).

Fellows. P.; Tecnología del procesado de los alimentos. (Principios y prácticas), Editorial Acribia, S.A., Zaragoza-España, pp 343-352 (1994).

Geankoplis, C.J.; Transport Process and Unit Operation. $3^{\mathrm{a}}$ Edition, Prentice-Hall PTR, Englewood Cliffs, New Jersey. pp. 345 (1993).

Hubbard, J.L., y B.E. Farkas; A method for determining the convective heat transfer coefficient during immersion frying, Journal and Food Process Engineering: 22(2), 201-214 (2000a).

Hubbard, J.L. y B.E. Farkas; Influence of oil temperature on convective heat transfer during immersion frying, Journal of Food Processing Preservation: 24(2), 143-162 (2000b).

Minagricultura; Ministerio de Agricultura y Desarrollo Rural., Anuario estadístico del sector agropecuario. 2003. Bogotá. (2003). Disponible en: http://www.agrocadenas.gov.co/lacteos/ Documentos/caracterizacion lacteos.pdf. Acceso: 15 de febrero (2008).

Moreira, R.G., J.K. Palau, V.E. Sweat y X. Sun; Thermal and physical properties of tortilla chips as a function of frying time, Journal of Food Processing and Preservation: 19(4), 175-189 (1995).

Moreira, R.G., M.E. Castell-Perez y M.A. Barrufet; Deep- fat frying. Fundamentals and applications, An aspen publication, Gaithersburg, Maryland, p.p 75-177 (1999).

Sahin, S., S.K. Sastry y L. Bayindirli; Heat transfer during frying of potato slices, Journal of Food Engineering: 32(1), 19-24 (1999).

Singh, R.P.; Heat and mass transfer in foods during deep-fat frying, Food Technology: 49(4), 134-137 (1995).

Sosa-Morales, M.E., R. Orzuna-Espiritu y J.F. Velez-Ruiz; Mass, thermal and quality aspects of deepfat frying of pork meat, Journal of Food Engineering: 77(3), 731-738 (2006).

Rice, P. y M.H. Gamble; Technical note: Modelling moisture loss during potato slices frying, Journal of Food Science Technology: 24(2), 183-187. (1989).

Vélez, R.J.F. y M.E. Sosa-Morales; Heat and mass transfer during the frying process of donuts, by CRC Press LLC (2003).

Whitaker, S.; Simultaneous heat, mass and momentum transfer in morous media: a theory of drying. In Advances in Heat Transfer: 13, 119-203 (1977)

Yildiz, A.T., K. Palazoglu y F. Erdogdu; Determination of heat and mass transfer parameters during frying of potato slices, Journal of Food Engineering: 79(1), 11-17 (2007). 
